\title{
A PSICOLOGIA E A ESTRATÉGIA SAÚDE DA FAMÍLIA: COMPONDO SABERES E FAZERES
}

\author{
Celiane Camargo-Borges \\ Cármen Lúcia Cardoso \\ USP - Ribeirão Preto
}

\begin{abstract}
RESUMO: Este ensaio traz para o debate possíveis articulações entre a psicologia e a Estratégia Saúde da Família (ESF). O texto inicia com um recorte histórico das transformações ocorridas na área da saúde culminando na implantação do SUS e na inserção de outros profissionais na Saúde Pública. Destaque especial se dá a psicologia, principalmente no campo da Psicologia Social da Saúde, que propõe uma atuação mais social, interativa, coletiva e local. A ESF, política pública instaurada para auxiliar na reorganização do sistema de saúde e na consolidação do SUS, ressalta a inter-relação de profissionais da saúde/usuários enfatizando a produção de vínculos, a criação de laços de compromisso e a co-responsabilidade. Por fim, o esforço é de articular os saberes e fazeres de cada campo, apontando essa composição como fértil no sentido de contribuir com a produção do cuidado em saúde e, ainda, com a reorientação do modelo assistencial.

Palavras-chave: Estratégia Saúde da Família; psicologia social da saúde; Modelo assistencial em saúde.
\end{abstract}

\section{PSYCHOLOGY AND THE FAMILY HEALTH CARE STRATEGY: COMBINING KNOWLEDGES AND PRACTICES}

ABSTRACT:This essay brings to discussion the links between Psychology and the Family Health Care Strategy (ESF). The text begins with a historical view of the transformations occurred in the health field leading to the National Public Health (SUS) and the participation of other professionals in the Public Health field. Special outstanding is given to Psychology, specially health social psychology which proposes an intervention more social, interactive, collective and local contexts. The ESF, a public policy that was established to promote the reorganization of the Health System and the consolidation of the SUS, emphasizes the relationship between health professionals / patients and the production of links, the creation of compromise and co-responsibility among them. Finally, the effort is to articulate the knowledges and practices of each field, pointing to the usefullness of this combination to health care work, and also, to the reorganization of assistance model. Key-words: Family Health Care Strategy; Health social psychology; Health assistance model

... E há que se cuidar do broto para que a vida nos dê flor.... (Milton Nascimento)

Para que a vida nos dê flor é preciso cuidado diário, atento e apaixonado e, ainda, que se estabeleça uma relação de responsabilidade e compromisso entre as partes envolvidas nesse processo.

Desde a conquista legal de um Sistema Único de Saúde (SUS) para o País que legitimou a saúde como um processo dinâmico e complexo, especial atenção se tem dado a produção do cuidado à saúde, em dois aspectos principais: o processo de trabalho e a integralidade. A produção do cuidado é compreendida como parte integrante da transformação do processo de trabalho em saúde, auxilia a estruturação das ações que abrangem as questões micro-políticas do trabalho, está atenta às necessidades em saúde no que diz respeito a vertentes locais, subjetivas e relacionais. Busca a integralidade da atenção, ou seja, o envolvimento responsável e compromissado de todas as partes implicadas nesse processo do cuidar - profissionais, usuários, organizadores e prestadores de serviços (Merhy, 2002; Merhy, 1997; Merhy, 1998).

O Programa de Saúde da Família (PSF) tem sido atualmente compreendido, pelo Governo Federal, como uma política pública de atenção primária à saúde (APS), sendo o primeiro contato da população com o serviço de saúde e uma estratégia para reorientação do sistema de saúde, auxiliando a operacionalização dos princípios e diretrizes do SUS e organizando o sistema numa rede articulada com os outros níveis de atenção (Brasil, 1997). Assim, o Programa tem sido denominado, mais recentemente, de Estratégia, e constitui a ampliação da perspectiva de um programa de ações em saúde, para que fique explicita a proposta de uma forma de reorganizar o modelo de atenção que visa a integralidade.

Na fomentação de uma nova política pública 
de saúde, abrem-se espaços de trabalho para a psicologia, que passa a problematizar a aplicação das práticas tradicionais em novo cenário de atuação. Outras ferramentas de intervenção - mais apropriadas para a efetiva inserção na área - devem ser construídas para o trabalho na Saúde Pública, para que possam contribuir para as transformações propostas pelo SUS. A Psicologia Social da Saúde, que compreende, em seus pressupostos, uma intervenção mais local e coletiva, tem sido um importante campo de conhecimento e prática para construir formas diferenciadas de intervenção na saúde.

O presente trabalho pretende contribuir para a reflexão sobre esses acontecimentos na saúde e como a psicologia se insere em tal sistema. É proposto aqui, uma composição de saberes e fazeres, com a articulação da Psicologia Social da Saúde e a ESF, apostando na potência dessa composição para a transformação da produção do cuidado em saúde. Este estudo visa, ainda, contribuir para a perspectiva da mudança que vem acontecendo na área, na busca de uma assistência menos tecnicista, mais humanizada, e parafraseando Milton, que juntos consigamos promover essas transformações e produzir o cuidado necessário "para que vida nos dê saúde".

\section{DA RECONFIGURAÇÃO DO SISTEMA DE SAÚDE À ESTRATÉGIA DE SAÚDE DA FAMÍLIA (ESF)}

Nas décadas de 70/80, acontecimentos nos âmbitos: social, político e econômico acarretaram profundas transformações na sociedade brasileira. Um processo crescente de endividamento externo, seguido de um intenso questionamento do regime político autoritário que governava o país, começou a enfraquecer o governo e a incentivar movimentos sociais de diversas ordens que buscavam melhores condições de vida.

Nesse contexto, na área da saúde, o fracasso do modelo prestador de serviços, a privatização da assistência médica, a crise financeira da previdência social, os altos índices de desemprego, um perfil epidemiológico marcado por altas taxas de mortalidade materna e infantil, o aumento das doenças infecto-contagiosas e os altos índices de acidentes de trabalho geraram uma grande mobilização social que ficou conhecida como movimento sanitário (Dimenstein, 1998).

Esse movimento trouxe propostas de novas concepções do pensar e fazer saúde - mais humana e universal - que só poderia ser alcançada através de uma ampla reforma sanitária. Seu caráter ideológico inicial foi se convertendo em outro mais pragmático, construiu alternativas concretas para reformulação do sistema de saúde e contribuiu para o processo de democratização do País (Teixeira \& Mendonça, 1989).
A VIII Conferência Nacional de Saúde, em 1986, foi o espaço da problematização do conceito de saúde visto como ausência de doenças e questionou o foco nos aspectos biológicos, num esforço de redefinição para uma concepção mais ampliada e dinâmica - a saúde como produto social resultante da ação de diversos determinantes: acesso a lazer, escola, saneamento básico, trabalho, serviços de saúde, entre outros. (Conferência Nacional de Saúde, 1986; Mendes, 1996).

A partir das propostas da VIII Conferência, a saúde foi incluída na Constituição do Brasil de 1988, no capítulo da Seguridade Social, como um direito de todos e dever do Estado, o que representou um avanço em relação à Constituição anterior.

Em setembro de 1990, foi regulamentada a reforma sanitária com a proposição do Sistema Único de Saúde (SUS), através da homologação da Lei Orgânica da Saúde que vigora no país atualmente (Cordeiro, 1991).

Os princípios do SUS são: universalidade no atendimento; eqüidade; integralidade nas ações em saúde; regionalização e hierarquização das unidades prestadoras de serviços; fortalecimento dos municípios; descentralização da gestão administrativa; resolutividade e participação popular.

Nesse contexto de mudanças oficializou-se, em 1994, o Programa de Saúde da Família (PSF). As ações do PSF foram, inicialmente, implantadas em regiões de escassa assistência à população, com os objetivos de atender às minorias sem acesso a serviços de saúde e de responder a uma tendência mundial de redução de custos, de desmedicalização da medicina e humanização dos serviços (Vasconcellos, 1998). Por esse programa inicial ser considerado potente para a universalização do atendimento a saúde e para implementar os preceitos da reforma sanitária brasileira, passou-se a haver um esforço e um incentivo para que se transformasse em Estratégia (ESF) de um projeto único do sistema da saúde e responsável pela APS.

A ESF visa estruturar o modelo de atenção voltado para a integralidade e a qualidade da assistência prestada e contribuir para consolidação dos princípios e diretrizes do SUS (Chagas e Seclen, 2003).

A ESF incorpora e reafirma os princípios do SUS, com as especificidades de um trabalho de APS. Com a proposta de ser a porta de entrada do usuário no sistema, a ESF se localiza num território específico, composto de 600 a 1000 famílias a serem acompanhadas por uma equipe mínima, integrada por profissionais médicos, enfermeiros, auxiliares de enfermagem e agentes comunitários de saúde. A equipe de saúde bucal foi recentemente integrada à ESF e é com- 
posta por um cirurgião dentista, um atendente de consultório dentário e um técnico em higiene dental (Brasil, 2002).

A idéia de porta de entrada visa ser não somente o primeiro acesso da população ao serviço, mas também um dispositivo de responsabilização institucional e sanitária no processo do cuidado com a saúde articulado a toda a rede de serviços (Brasil, 2003). Segundo Ciampone e Peduzzi (2000), um dos pontos centrais do trabalho da ESF seria "o estabelecimento de vínculos e a criação de laços de compromisso e de co-responsabilidade entre os profissionais da saúde e a população" (p.143), numa tentativa de romper com a perspectiva tecnicista em saúde e fortalecer o envolvimento dos atores sociais no processo saúde-doença-cuidado.

Os fundamentos da ESF - inserção num território específico, além do espaço geofísico, mas também espaço da cultura, das relações, trabalho local com a população, com estabelecimento de vínculos, produção de acolhimento e responsabilização aproximam o profissional e suas ferramentas de ação ao âmbito da micro-política dos processos de trabalho, nos seus fazeres cotidianos, nas suas relações, seja com outros profissionais seja com a comunidade. Portanto, trabalhar em consonância com a proposta da ESF requer uma inversão da lógica do cuidado menos técnico e mais relacional - tanto entre equipeusuário como entre equipe-equipe (Camargo-Borges, 2002).

De um enfoque biomédico e disciplinar tradicional, a mudança na saúde trouxe alguns ruídos sobre outras formas de atuação no sistema, principalmente na ESF, que tem seu objeto de trabalho diferenciado pela atuação direta com a comunidade, em seu cotidiano. A conduta profissional, nessa forma de trabalho, passa a ser mais coletiva. Enfoca a família e suas relações e não mais somente o indivíduo com seus problemas e resgata as múltiplas dimensões da saúde, o que exige o reformular da postura de intervenção do profissional, assim como a incorporação de outros saberes para compor a produção do cuidado com a saúde.

Nesse contexto, a psicologia dispõe de ferramentas que poderão ser úteis para a construção de um modelo mais integrado e holístico de atenção à saúde.

\section{A PSICOLOGIA NO CAMPO DA SAÚDE PÚBLICA}

Até a década de 70, a participação da psicologia no âmbito da saúde pública ainda era incipiente.

A psicologia, enquanto profissão tem uma história recente no Brasil. Apoiada na regulamentação de 1962, através da Lei Federal 4.119, tradicionalmente foram constituídas quatro áreas de atuação do psicólogo: a clínica, a escolar, a industrial e o magistério. A psicologia, desde a sua criação, é reconhecida como profissão liberal - parecer 403/68 (Lei 4.119). A ênfase das atividades deste profissional se centrou nas décadas seguintes, no trabalho autônomo, clínico, individual, curativo e voltado para uma clientela financeiramente privilegiada no acesso. (Silva, 1992; Dimenstein, 1998).

Na reconfiguração do sistema de saúde, o psicólogo passa a integrar equipes. Entretanto, não dispunha de um arcabouço teórico e prático para atuação nesse âmbito, o que, muitas vezes, contribuiu para a manutenção do modelo médico hegemônico, do trabalho com enfoque no tratamento de fenômenos da esfera psíquica ou mental sem necessidade de compreendê-los a partir de suas multideterminações, ou seja, sem considerar o contexto social, econômico e político no qual o indivíduo está imerso (Spink, 1992; Silva, 1992; Dimenstein, 1998).

A psicologia, num processo de revisão dessas práticas e busca por melhores formas de responder às necessidades dos diferentes locais de atuação, foi gerando novos campos de saber e ampliou sua inserção na saúde.

A Psicologia Social da Saúde configura-se como um campo de conhecimento e prática que trata das questões psicológicas com enfoque mais social, coletivo e comunitário voltado para a saúde. Segundo Marín (1995), caracteriza-se pela interlocução da psicologia Social - com seus conhecimentos e técnicas - com o âmbito da saúde e destaca a interação como ponto fundamental do processo saúde-doença. A interação refere-se tanto ao homem e seu ambiente quanto aos diversos atores sociais presentes no cuidado à saúde. $\mathrm{O}$ autor ainda salienta que todas as atividades da Psicologia Social da Saúde centram-se mais na busca de uma saúde integral e não somente na saúde mental.

Spink (2003) trata da Psicologia Social da Saúde como um campo ampliado de atuação do psicólogo nas instituições de saúde. Essa ampliação ocorreria principalmente, em relação ao referencial de trabalho utilizado e exercido, e abrangeria duas principais questões que a autora destaca como fundamentais. Primeiramente, a questão contextual da intervenção, isto é, a importância de se compreender toda a história e o contexto da instituição na qual será implementada uma ação, assim como as pessoas que compõem essa instituição. Faz-se necessário compreender que cada organização tem sua realidade local, sua cultura de relações e as histórias específicas das pessoas que recorrem a esses serviços. A segunda enfatiza a questão do "outro", da alteridade. As intervenções nessa abordagem levam em conta a interface da cultura e do social no processo de construção da identidade e da inserção da pessoa 
na vida. É a percepção da construção dessa identidade, tão multiforme, que possibilita reconhecer a alteridade e lidar com o diverso. A alteridade é entendida como o relacionar-se com o outro, diferente de mim, mas reconhecido por mim como uma pessoa com direitos iguais aos meus e valorizada enquanto sujeito.

A Psicologia Social da Saúde, segundo Spink (2003), tem como características principais à atuação centrada em uma perspectiva coletiva e o comprometimento com os direitos sociais e com a cidadania. Rompe, portanto, enfoques mais tradicionais centrados no indivíduo. A atuação se dá principalmente nos serviços de atenção primária à saúde, focaliza a prevenção da doença e a promoção da saúde e incentiva os atores sociais envolvidos para a geração de propostas de transformação do ambiente em que vivem. Trata-se, portanto de um processo de transformação crítica e democrática que potencializa e fortalece a qualidade de vida.

Como proposição geral, a Psicologia Social da Saúde, ao contribuir para a superação do modelo biomédico, objetiva trabalhar dentro de um modelo mais integrado, reconhece a saúde como um fenômeno multidimensional em que interagem aspectos biológicos, psicológicos e sociais e caminha para uma compreensão mais holística do processo saúde-doençacuidado.

Dessa maneira sua inserção na ESF pode ser útil para contribuir para a transformação das práticas em saúde rumo à integralidade.

\section{A ESTRATÉGIA SAÚDE DA FAMÍLIA E A PSICOLOGIA SOCIAL DA SAÚDE: CONSTRUINDO PONTES}

Pensar a psicologia como parceira da ESF é um convite a pôr em pauta os processos de trabalho em saúde que acontecem no território da ESF e as relações que nele transcorrem. Um convite à reflexão e a problematização das possíveis formas de intervenção local, numa postura criativa para a composição de novos trabalhos, mais consoantes com cada realidade local.

Tal parceria pode contribuir para o esforço que tem sido feito para romper com o modelo biomédico que, até há muito pouco tempo, conduzia as ações em saúde e se desdobravam em formas especialistas e técnicas de trabalho, dificultando a integralidade dessas ações. Um exemplo dessas mudanças é a crescente demanda por trabalhos em equipes multiprofissionais.

Apesar de a Saúde Pública já pressupor um trabalho de equipe, resquícios dessa forma de intervenção ainda dificultam um trabalho mais integrado. Segundo Peduzzi (2001), a equipe de saúde muitas vezes se organiza sem um agir comunicativo, que marca as relações hierárquicas de subordinação estabelecidas, valor comum atribuído ao modelo biomédico tradicional de saúde. A equipe multidisciplinar que tem como proposta constituir-se como um espaço para a dialogia e para a troca de saberes, muitas vezes tem sido utilizada para o estabelecimento de divisão de trabalho, de papéis, fortalecendo a individualização dos profissionais em detrimento de relações mais horizontais e coletivas. Desse modo, a possibilidade de uma intervenção diferenciada, de um trabalho integrado, intersetorial, voltado para a coletividade, muitas vezes se torna difícil operacionalizar. Prevalece, assim, a dicotomia entre a competência técnica e a sensibilidade social.

Constata-se que a psicologia cada vez mais tem adentrado o terreno da saúde, incrementando a compreensão do processo saúde-doença-cuidado, principalmente na área da saúde mental, tendo crescido, quantitativamente, em hospitais e postos de saúde. Na ESF, a psicologia tem despontado através da proposição de algumas intervenções, já consagradas, no âmbito da saúde, como o trabalho com grupos (Souza \& Carvalho, 2003; Cardoso, 2002) e o das equipes de saúde mental (Lancetti, 2003). A intervenção através de grupos na ESF acontece, principalmente, com os chamados grupos programáticos para o cuidado de questões prevalentes na saúde, tais como os grupos de hipertensão e diabetes e os de gestantes. Esses grupos têm, geralmente, caráter informativo e número determinado de encontros. Já o trabalho da saúde mental, na ESF, tem sido constituído por profissionais dessa área (psicólogos e psiquiatras) que assessoram a equipe mínima através de estudo de casos, interconsultas, supervisão continuada, orientação e capacitação no cuidado e acolhimento dos casos de saúde mental (Lancetti, 2003).

Porém, o que está se problematizando nessa reflexão é a conformação de um novo saber que seja sensível a múltiplos contextos; busca-se a produção de diferentes conhecimentos para ampliação do foco de trabalho, para constituir laços diferenciados de interação entre as diversas áreas da saúde.

A Psicologia Social da Saúde traz conceitos potentes e propostas de ação que muito se aproximam dos pressupostos de trabalho da ESF. Vemos que os dois discursos se organizam em torno de eixos que apostam na construção do fazer conjunto, coletivo e valorizam a localidade e as interações dela decorrentes.

A forma como a ESF está organizada, localizada num território, trabalhando em equipe, focalizando o sistema familiar, a cultura local e lidando diretamente com o cotidiano das pessoas, a faz deparar com questões que demandam acolhimento, vínculo, interação e são da ordem da imprevisibilidade, pois, a cada encontro, a cada situação, gera-se uma 
forma diferente de intervenção. Segundo Sousa (2003) o território na ESF é caracterizado como "sinônimo de lugar, espaço de interação, solidariedade" (p. 34). Algumas diretrizes são postas para tentar operacionalizar esse tipo de organização, como a longitudinalidade e o planejamento ascendente.

A longitudinalidade é definida como o segmento do usuário e de sua família no tempo, com a formação de vínculo, independente da presença de patologia.

O planejamento ascendente é definido como o delineamento de estratégias de intervenção nas famílias, de acordo com as necessidades da comunidade que reside no território.

Os pressupostos da Psicologia Social da Saúde ecoam, nesse modo de organização do trabalho, à medida que têm como ponto fundamental também a contextualidade e a interação com ações construídas coletivamente a partir das imprevisibilidades do cotidiano. Assim, tanto a ESF como esse campo da psicologia privilegiam o processo de produção de conhecimento e a construção das intervenções a partir das práticas sociais, dos processos interativos e da cultura. A proposta, portanto, é a de que qualquer entendimento do processo saúde-doença-cuidado possa ser analisado e referido a partir de seu contexto, ou seja, a partir da compreensão de uma pessoa, pertencente a determinada família, inserida numa comunidade específica, e assim por diante.

A Organização Panamericana de Saúde enfatiza que a ESF deva ser constituída a partir do seu contínuo fazer e refazer. Este "fazer-fazendo" também é compartilhado pela Psicologia Social da Saúde, já que suas intervenções caracterizam-se por um construir e reconstruir coletivos, com pessoas imersas nessa rede de sentidos (re) negociando-os o tempo todo.

A produção do vínculo nas relações de trabalho também é foco tanto da ESF quanto da Psicologia Social da Saúde. Na ESF, o acolhimento é realçado como peça fundamental. $\mathrm{O}$ acolhimento é uma forma de intervenção que propõe apoio contínuo à pessoa em todo o seu processo de atendimento na saúde e não somente no que diz respeito ao acesso do usuário ao serviço. Abrange o encontro do profissional com esse usuário, num processo de negociação das necessidades deste, promovendo acesso, buscando a produção do vínculo (Matumoto, 1998). A Psicologia Social da Saúde se interessa pela criação de práticas conversacionais que possam construir caminhos possíveis para que as pessoas sigam juntas mesmo na diversidade, ou seja, com vínculos entre elas. Interessase pela utilização de um discurso que possa ser compartilhado, para que se criem mais possibilidades de ação. Tais práticas estão dizendo que o acolhimento e o vínculo são potenciais na criação de um espaço coletivo de atuação. O estabelecimento do vínculo na relação, segundo Campos (1997), promove melhor eficácia das ações em saúde e facilita a participação do usuário nestas ações.

Por fim, como já visto, as propostas do SUS de democratização da saúde e de inversão da lógica hierárquica e autoritária que tradicionalmente vinha funcionando no sistema têm como ponto fundamental a ESF, objetivando a criação de uma rede integrada de cuidados progressivos. A interlocução com a Psicologia Social da Saúde ocorre no suporte para a construção de espaços mais democráticos de convivência, no trato à alteridade, propiciando conversas mais igualitárias, promovendo integração entre profissionais - usuários e, com isso, contribuindo para a eficácia do sistema de saúde idealizado pelo SUS - universal, eqüitativo, integral.

Assim, a Psicologia Social da Saúde viria ao encontro desse desafio da ESF em construir um modelo de atenção à saúde pertinente à realidade local e gerador de interlocuções entre equipe de saúde e comunidade. Nesse sentido, a parceria pode ser útil para pensar discursos, na saúde, que propiciem a construção de espaços viabilizadores de acolhimento e a construção do vínculo, contribuindo para a reflexão e a problematização dessas práticas que se propõem coletivas.

Tal parceria investiria no fortalecimento das relações locais, propondo-se a reconhecer e legitimar a diversidade nesses espaços de encontro e resgatando a cidadania dos atores sociais envolvidos.

A articulação da Psicologia Social da Saúde com a ESF apresenta-se como proposta que tem muito a contribuir para ações junto às comunidades, com o objetivo de compor e não substituir. Compor um trabalho em que a equipe interaja e funcione como potência para o território da intervenção, com a criação de sistemas conversacionais na equipe que se multipliquem em diálogo para toda a comunidade, como estratégia permanente de ação. Construir um projeto institucional abraçado pela equipe como um todo, numa abertura para a diversidade, com alternativas que possam traduzir-se em co-responsabilidade, tão almejada no sistema de saúde.

A proposta do presente trabalho não é, de maneira alguma, a de levantar a bandeira de uma luta corporativa de modo a garantir inclusão de mais um especialista na equipe da ESF. Não se tem aqui o propósito de dar continuidade à segmentação da saúde, em que cada "pedaço" seria de responsabilidade de um especialista específico, cada âmbito pertenceria à competência de um profissional. Isso seria avesso à fundamentação da ESF que prega o rompimento com os paradigmas do modelo anterior e busca a integralidade; o fato descaracterizaria toda a argu- 
mentação que se procura construir neste ensaio.

O objetivo dessa reflexão é ampliar! Discutir possibilidades de criação de propostas que possam ser potentes para as práticas que a ESF pretende instaurar em sua lógica de trabalho. A psicologia, como disciplina que tem em seu objeto de estudo as relações humanas, as interações, os afetos, pode trazer contribuições para tal tarefa de construção dessa Estratégia do sistema de saúde que vem tentando se pautar por práticas mais relacionais. Especificamente, a Psicologia Social da Saúde que, como vimos, faz pontes conceituais com a ESF, pode auxiliar o embasamento e o suporte de ações construídas nesse contínuo "fazer-fazendo" que, ideologicamente, constitui o projeto de intervenção da ESF.

\section{REFERÊNCIAS}

Brasil. Ministério da Saúde. (2003). O desenvolvimento do Sistema Único de Saúde: avanços, desafios e reafirmação dos seus princípios e diretrizes. Brasília.

Brasil. Ministério da Saúde. (2002). Guia Prático do Programa de Saúde da Família. Brasília.

Brasil. Ministério da Saúde. (1997). Saúde da família: uma estratégia para a reorganização do modelo assistencial. Secretaria de Assistência à Saúde. Brasília.

Camargo-Borges, C. (2002). Sentidos de saúde/doença produzidos em grupo numa comunidade alvo do Programa de Saúde da Família (PSF). 161 f. Dissertação (Mestrado). Faculdade de Filosofia Ciências e Letras, Universidade de São Paulo, Ribeirão Preto.

Cardoso, C.L. (2002). A inserção do psicólogo no Programa de Saúde da Família. Psicologia, Ciência e Profissão (pp. 2-9). São Paulo, n. 22.

Campos, G.W.S. (1997). Considerações sobre a arte e a ciência da mudança: revolução das coisas e reforma das pessoas. O caso da saúde. In: Cecílio, L. C. O. (Org.). Inventando a mudança na saúde (pp. 29-87). São Paulo: Hucitec.

Chagas, L.; Seclen, J. (2003). Discussão sobre o marco referencial e conceitual da Atenção Básica e Saúde da Família no Brasil. In: Organização Panamericana de Saúde-OPAS. Projeto de desenvolvimento de Sistema e Serviços de Saúde. Brasília.

Ciampone, M.H.T.; Peduzzi, M. (2000). Trabalho em equipe e trabalho em grupo no Programa de Saúde da Família. Revista Brasileira de Enfermagem (pp. 143-
147). Brasília, v. 53, n. especial.

Conferência Nacional De Saúde. (1986). Anais da VIII Conferência Nacional de Saúde. Brasília.

Cordeiro, H. (1991). Sistema Único de Saúde. Rio de Janeiro: Ayuri Editorial.

Dimenstein, M. D. B. (1998). O Psicólogo no contexto do Sistema Único de Saúde (SUS): Perfil profissional e perspectivas de atuação nas unidades básicas de saúde. (UBS). 223f. Tese (Doutorado). Universidade Federal do Rio de Janeiro, Rio de Janeiro.

Lancetti, A. (Org.) (2003). SaúdeLoucura 7. Saúde mental e saúde da família. São Paulo: Hucitec.

Marín, J. R. (1995). Psicologia social de la salud. Madri: Editorial Sínteses.

Matumoto, S. (1998). O acolhimento: um estudo sobre seus componentes e sua produção em uma unidade da rede básica de serviços de saúde. 1998. 187f. Dissertação (Mestrado), Escola de Enfermagem de Ribeirão Preto, Universidade de São Paulo, Ribeirão Preto.

Mendes, E.V. (1996). Um novo paradigma sanitário: a produção social da saúde. In: Uma agenda para a saúde. (pp.233-298). São Paulo: Hucitec.

Merhy, E.E. (2002). Saúde, a cartografia do trabalho vivo. São Paulo: Hucitec.

Merhy, E.E. (1997). Em busca do tempo perdido: a micro-política do trabalho vivo em saúde. In: Merhy, E. E.; Onocko, R. (Orgs). Agir em Saúde um desafio para o público. (pp. 71-112). São Paulo: Hucitec; Buenos Aires: Lugar Editorial.

Merhy, E.E. (1998). A perda da dimensão cuidadora na produção da saúde - Uma discussão do modelo assistencial e da intervenção no seu modo de trabalhar a assistência. In: Reis, A. T.; Santos, A. F.; Campos, C. R.; Malta, D.C.; Merhy, E. E. (Orgs) Sistema Único de Saúde em Belo Horizonte - Reescrevendo o público (pp.103-120). São Paulo: Xamã.

Peduzzi, M. (2001). Equipe multiprofissional de saúde: conceito e tipologia. Revista de Saúde Pública (pp. 103-109). São Paulo, v.35, n.1.

Silva, R.C. (1992). A formação em psicologia para o trabalho na saúde pública. In: Campos, F. C. B Psicologia e saúde: repensando práticas (pp. 25-40). São 
Paulo: Editora Hucitec.

Sousa, M. F. De. (2003). A cor-agem do PSF. 2. ed. São Paulo: Hucitec.

Souza, R. A.; Carvalho, A. M. (2003). Programa de Saúde da Família e qualidade de vida: um olhar da psicologia. Estudos de psicologia (pp.515-523). Natal, v.8 n.3.

Spink, M. J. (2003). Os Psicólogos na saúde Reflexões sobre os contextos da prática profissional. In: Psicologia social e saúde: práticas, saberes e sentidos (pp.77-159). Petrópolis: Editora Vozes.

Spink, M. J. (1992). Psicologia da saúde: A estruturação de um novo campo de saber. In: Campos, F. C. B Psicologia e saúde: repensando práticas (pp. 11-23). São Paulo: Editora Hucitec.

Teixeira, S. F; Mendonça, M.H. (1989). Reformas Sanitárias na Itália e no Brasil: comparações. In: Teixeira, S.F. (Org). Reforma Sanitária em busca de uma teoria (pp. 193-232). São Paulo: Cortez. Associação Brasileira de Pós-Graduação em Saúde Coletiva.

Vasconcellos, M. da P. C. (1998). Reflexões sobre a Saúde da Família. In: Mendes, E. V. (org), A organização da saúde no nível local. (pp. 155-172). São Paulo: Hucitec.

Celiane Camargo-Borges é Mestre em Psicologia e Doutoranda em Saúde Pública - Escola de Enfermagem de Ribeirão Preto -Universidade de São Paulo. O endereço eletrônico da autora é: celianeborges@uol.com.br

Cármen Lúcia Cardoso é Professora doutora do Departamento de Psicologia e Educação da Faculdade de Filosofia, Ciências e Letras de Ribeirão Preto - Universidade de São Paulo. Endereço para correspondência: FFCLRP -USP. Depto. de Educação. Av. Bandeirantes, 3900 CEP 14040-901. Ribeirão Preto - SP

O endereço eletrônico da autora é: carmen@ffclrp.usp.br

Celiane Camargo Borges

Carmén Lúcia Cardoso

A psicologia e a estratégia saúde da família: compondo saberes e fazeres

Recebido:22/03/2005

$1^{\text {a }}$ revisão: 27/06/2005

Aceite final: 25/08/2005 